\title{
BARBARIE, ÉMOTION ET ALTÉRITÉ : LES AFFECTS « EXCESSIFS » DE LA SLAVITÉ FIN-DE-SIĖCLE DANS LES NORONSOFF DE JEAN LORRAIN
}

\author{
CÉCILE GAUTHIER \\ Université de Reims Champagne-Ardenne
}

\section{BARBARY, EMOTION AND ALTERITY: SLAVIC "EXCESSIVE AFFECTS" IN JEAN LORRAIN'S LES NORONSOFF (1902)}

\begin{abstract}
Amidst all the collective emotions prevailing in $19^{\text {th }}$ century France, a remarkable one is fear, which can be perceived in the myth of Barbarian invasions. The opposition between the so-called "civilized" and "barbarian" men is rooted in the emotional excess and disorders which are thought to affect the latter. In Jean Lorrain's Les Noronsoff (1902) a Russian character, whose excessive actions are both comical and pathetic, embodies fin-de-siècle barbary. In the racialist ideology of that time, Slavic people were said to lack a stable nature and to tend to imitation and metamorphosis. This choice of a Slavic character illustrates the variation between (civilized) identity and (barbarian) alterity. Slavic barbary, which combines the two faces of barbary (interior and exterior), reveals both the fear and the fascination raised when contemplating emotional flows spreading without control, and reflecting one's own identity.
\end{abstract}

Keywords: French Literature, Jean Lorrain, Barbary, Slavs, Russians Mots clés : Littérature française, Jean Lorrain, barbarie, slavité, Russes

Le XIX ${ }^{e}$ siècle français, envisagé dans la perspective d'une histoire des émotions, oscille entre l'héritage des grandes émotions collectives de la Révolution française et de l'Empire, et le repli sur l'intime, historicisé par les romantiques qui y voient une conséquence du contexte politique ${ }^{1}$. L'une de ces émotions, la peur, adopte, entre autres, la forme mythique des invasions barbares. Le socialiste Pierre Leroux qualifie cette peur de " grand cheval de la contre-révolution ${ }^{2}$ ". Ce mythe a donc à voir avec l'excès d'émotions, et met au jour des tensions contradictoires dans l'idéal de civilisation : en effet la part belle est faite à la raison et à l'idéologie du progrès, héritée du XVIII siècle où s'est imposée l'opposition entre civilisation et barbarie. La taxinomie rationalisante de la science assigne à chaque objet une place déterminée, tandis que, dans l'univers bourgeois qui

1 Voir Corbin, A. - Courtine, J.-J. - Vigarello G. (2016) : Histoire des émotions. Volume 2. Des Lumières à la fin du XIXe siècle. Paris : Seuil ; et notamment l'article de Judith Lyon-Caen, «Le "je" et le baromètre de l'âme", pp. 169-188.

2 Cité dans l’article « Barbares » du Dictionnaire du romantisme (2012). Paris : CNRS Editions, p. 64. 
triomphe socialement, l'émotion est reconnue mais contenue dans l'intimité domestique. Il semble donc bien y avoir une tendance à la domestication des émotions, au refus des débordements. Mais ce n'est peut-être que le revers du flux émotionnel qui traverse ce siècle de bouleversements politiques, socio-économiques et épistémologiques. Le culte du progrès ne doit ainsi pas faire oublier que la fascination pour l'irrationnel court tout au long du siècle, notamment dans le champ artistique, du romantisme au symbolisme. "Expression d'une crise de la civilisation ${ }^{3}$ ", le mythe des invasions barbares serait une des formes, toute de contradictions, prises par ce questionnement identitaire dans les sociétés européennes en mutation. En effet ce mythe conduit dans le même temps à ériger et défaire la polarité entre identité et altérité, ou « civilisés » et « barbares » : si le désordre émotionnel, dans l'imaginaire collectif, est présenté comme le fait des barbares, il ne semble de fait pas étranger aux " civilisés ", eux-mêmes sujets à des hantises reflétées ou reconfigurées par l'art.

Nous nous arrêterons dans cet article sur la réactualisation de ce mythe des invasions barbares dans l'imaginaire fin-de-siècle, tel qu'il transparait dans l'œuvre de Jean Lorrain (1855-1906). Dans les dernières décennies du XIX ${ }^{\mathrm{e}}$ siècle, la France, fragilisée par la défaite contre la Prusse, est à la fois secouée par des tensions politiques liées à l'établissement de la République, toujours objet de résistances, et par le durcissement de l'antisémitisme dont témoigne l'affaire Dreyfus. Dans ce contexte resurgit le mythe de l'envahisseur venu de l'Est, qui a parcouru tout le siècle : un des visages de la barbarie dans l'imaginaire collectif est en effet celui de la slavité, parfois croisée avec la judéité. Il vient réactiver l'image sidérante des « cosaques» défilant dans les rues de Paris en 1814-18154. Dans Les Noronsoff (1902), Jean Lorrain, lui-même qualifié par ses contemporains de " grand barbare ", décline sa version de la barbarie slave. Ce roman est marqué par l'excès monstrueux d'émotions, jusqu'à une forme pathologique. Nous nous interrogerons sur le sens de ce choix de la slavité pour incarner, avec humour et provocation, un déséquilibre émotionnel qui s'avère autant extérieur qu'intérieur à l'homme « civilisé ».

\section{« Barbares » et désordre des émotions}

On sait que le barbare désigne à l’origine l'étranger, le non-grec, non-romain, celui qui est différent ethniquement, culturellement, et linguistiquement. Roger Pol-Droit, dans sa Généalogie des barbares, déconstruit un certain nombre d'idées reçues : il rappelle que, si les barbares évoqués dans les textes antiques peuvent se comporter avec cruauté et férocité (donc de façon «barbare », selon les usages ultérieurs du mot), cela n'est pas systématique : il y a même des barbares reconnus comme philosophes, détenteurs d'une sagesse antérieure à la pensée grecque - par exemple en Égypte ${ }^{5}$. Roger Pol-Droit insiste en outre sur la distinction à opérer entre «barbare » et «barbarie », ce dernier mot n’étant

3 Brogniez, L. (2001) : « Le mythe de l'invention des barbares en Belgique : art ou/et révolution au tournant du siècle ». In Montandon, A. Mythes de la décadence. Clermont-Ferrand : Presses Universitaires Blaise Pascal, pp. 160-161.

4 Voir les ouvrages de M.-P. Rey, notamment Un tsar à Paris. 1814. Alexandre I Ir et la chute de Napoléon (2015). Paris, Flammarion, «Champs », pp. 221-241.

5 Voir le chapitre 6 dans Pol-Droit, R. (2007) : Généalogie des barbares. Paris, Odile Jacob. 
pas utilisé par les Grecs. Sa thèse est la suivante : les barbares sans barbarie auraient disparu pour faire place à la barbarie sans barbares. Alors que chez les Grecs, on naissait barbare, du fait, principalement, d'une appartenance ethnique, aujourd'hui ce serait au terme d'un processus que l'homme serait susceptible de devenir barbare, en raison d'actes jugés inhumains par leur degré de cruauté. Les analyses du médiéviste Jean-Pierre Poly vont dans le même sens :

Le barbare [...] est coupé en deux : le barbare d'origine - ce sauvage européen - est « outrepassé » dans l'obscurité d'un temps jadis très lointain, livré à la futilité présumée des études historiques, en fin de compte rejeté à l'incohérence de sa qualification ancienne ; la barbarie en revanche, s'est détachée de lui pour devenir moderne. [...] Elle est devenue le mal suprême, la perversion contemporaine de notre être ${ }^{6}$.

Quel rapport entre les représentations des barbares et l'histoire des émotions ? On aurait tort de penser qu'est qualifié de «barbare » celui qui semble inhumain du fait de son absence d'émotions : l'étude des discours menée par Roger Pol-Droit montre que c'est plutôt un rapport désordonné à sa vie affective qui fait le barbare. Soit il souffre d'un excès d'émotions, passant sans contrôle d'un extrême à l'autre, soit il n'apparaît pas détenteur des émotions censées fonder l'humanité de l'homme selon un cadre défini par une philosophie, une religion. Il se caractérise donc par une dysharmonie émotionnelle, ainsi dans son rapport au logos. Héraclite par exemple qualifie de «barbare " (et ce sans spécificité ethnique) l'âme de ceux qui font du logos un usage insuffisant ${ }^{7}$. Pour Platon, le barbare échappe également à l'ordre de la raison : " Il apparaît comme l'homme de l'anarchie, de l'impulsivité, de l'émotivité incontrôlable et imprévisible. [...] Il cède à ses émotions comme à ses impulsions ${ }^{8}$ ". Les Romains cultivent eux aussi un goût (si ce n'est un devoir) pour la mesure dans la manifestation des émotions, qui constitue un des critères de partage entre les membres de la société romaine. Corbin, Courtine et Vigarello rappellent dans leur introduction à l'Histoire des émotions la célèbre citation de Sénèque dans sa Consolation à Marcia (VII, 3) : "Le même deuil affecte une femme plus qu'un homme, un barbare plus qu'une personne civilisée, un ignorant plus qu'une personne instruite 9 . " L'incapacité à être maître de soi est (cause ou conséquence ?) échue en partage aux « inférieurs ». Cet exemple illustre en outre ce que Roger Pol-Droit appelle la «fonction » du «barbare "- qui n'est pas pour lui un concept, mais un opérateur : " "barbare" a pour fonction de séparer un groupe et ses autres, de construire un dehors et un dedans ${ }^{10} »$. Or un bouleversement s'opère au XIX ${ }^{\mathrm{e}}$ siècle : les barbares auraient désormais franchi la ligne de partage.

\footnotetext{
6 Poly, J.-P. «Impérialisme et Barbarie ». In Guirlinger, L. - Poly, J.-P. - Lefort, C. - Mijolla-Mellor, S. de, Mattéi, J.-F. - Nahoum-Grappe, V. - Brossat, A. (2005) : La barbarie aujourd'hui, mythe et réalité. Nantes : Éditions Cécile Defaut, pp. 32-33.

7 Généalogie des barbares, op. cit., p. 41.

8 Ibid., p. 53.

9 Cité dans l'Histoire des émotions, Volume 1, op. cit., p. 9.

10 Généalogie des barbares, op. cit., p. 134.
} 


\section{L'intériorisation des barbares au XIXe siècle}

En quoi peut-on parler d'une intériorisation des barbares au XIX ${ }^{\mathrm{e}}$ siècle ? D'une part parce que la figure du barbare extérieur au monde civilisé et strictement délimitée par une ligne frontière ne serait désormais plus valide, du fait de la colonisation qui accroît le territoire possédé par les Européens au-delà de ses limites historiques, lui faisant ainsi absorber l'ancestrale barbarie. Et la faisant alors disparaitre ? Roger Pol-Droit estime de fait que les barbares « disparaissent, comme figures réelles ${ }^{11}$ »- pour resurgir avec d'autant plus de force dans l'imaginaire. Mais s'il y a disparition des "barbares », c'est surtout au sens où, désormais, les «barbares » ne constituent plus une altérité qui serait résolument extérieure, en dehors des limites imparties au monde civilisé. On remarque néanmoins que cette figure du barbare s'impose toujours davantage, non seulement dans l'imaginaire, mais comme objet du savoir anthropologico-linguistique, notamment dans le cadre de la formidable expansion des études " orientalistes ${ }^{12}$ ». Or celles-ci, dans leurs lignes de partage raciales, reconduisent une opposition entre raison et émotion (par exemple entre « race philosophique » indo-européenne et " race religieuse » sémite) qui n'est pas sans rappeler le partage entre « civilisés » et « barbares ", jusque dans la complexité d'une barbarie qui peut aussi contenir une sagesse archaïque. Les barbares seraient donc désormais certes beaucoup plus proches, cette proximité allant jusqu’à leur faire perdre leur statut de barbares pour acquérir celui de spécimens de l'espèce humaine, diverse, mais une. Néanmoins ils seraient en même temps tenus à distance par ce figement épistémologique enraciné dans une pensée biologique. C'est pourquoi le terme de « disparition » appelle à être précisé.

L'intériorisation des barbares tient d'autre part à l'émergence d'une barbarie « indigène ", d'un mythe barbare intrinsèque à l'Europe et éminemment romantique ${ }^{13}$. Elle découle de l'actualisation d'une antique et estimable barbarie redécouverte, ou plutôt réinventée, dans le cadre des constructions nationales et de la quête de racines auxquelles elle donne lieu. Les " races » supposées être à la source des nations modernes se voient ainsi remises à l'honneur par nombre de philologues, historiens, artistes et hommes politiques, si bien que les barbares, selon le mot d'Éric Michaud, se voient " débarbarisés ${ }^{14}$ ». Le mythe barbare est également politisé : les « barbares » du dedans, ce sont les hommes et femmes du peuple, ceux qui font désormais entendre leur voix, qui sont même susceptibles de violence - régénératrice ou destructrice. De fait les barbares, au sens ethnique ou au sens social, présentent le point commun dêtre porteurs d'une singulière énergie vitale. Politiquement, le mythe se voit interprété selon des axiologies radicalement opposées. Pour certains il traduit une peur des classes laborieuses comme classes « dangereuses ${ }^{15}$ ", ainsi dans cette phrase de Saint-Marc Girardin au moment de la révolte des Canuts en

11 Ibid., p. 245.

12 Nous renvoyons aux travaux d'Edward W. Said, qui a fait la démonstration de l'interconnexion des champs artistique, épistémologique et politique.

13 Voir Michel, P. (1981) : Un mythe romantique. Les Barbares 1789-1848. Lyon : Presses universitaires de Lyon.

14 Michaud, E. (2015) : Les Invasions barbares. Une généalogie de l'histoire de l’art. Paris : Gallimard, nrf essais, p. 115.

15 Selon la formule de Louis Chevalier, dans l'ouvrage classique (1958) : Classes laborieuses et classes dangereuses à Paris pendant la première moitié du XIXe siècle. Paris : Plon. 
1831 : «Les Barbares qui menacent la société ne sont point au Caucase ni dans les steppes de Tartarie : ils sont dans les faubourgs de nos villes manufacturières ${ }^{16}$. " On remarque que, même si le nom slave, encore peu usité en France ${ }^{17}$, n'apparaît pas, c'est bien la slavité qui est sous-jacente à cette désignation de la référence barbare par excellence. Michelet pour sa part, dans sa dédicace au Peuple (1846) renverse cette hantise d'un soulèvement des classes populaires en un geste de fière appropriation d'une nomination habituellement dégradante : "Souvent aujourd'hui l'on compare l'ascension du peuple, son progrès, à l'invasion des Barbares. Le mot me plaît, je l'accepte... Barbares ! Oui, c'est-à-dire pleins d'une sève nouvelle, vivante et rajeunissante ${ }^{18}$. " Le mythe des invasions barbares se métamorphose donc au gré des discours, et ne cesse de resurgir tout au long du siècle, se trouvant réactualisé dans des contextes politiques et culturels changeants. À la fin du siècle, il devient, dans le champ artistique, l'un des mythes de la décadence, dont s'empare un de ses plus scandaleux représentants, Jean Lorrain.

\section{Jean Lorrain, « grand barbare »}

Jean Lorrain occupa une place centrale dans la société parisienne de son temps : il fut un critique littéraire et chroniqueur redouté pour sa plume impitoyable, un dramaturge, poète et auteur de romans et nouvelles. Il incarne la décadence dans ses excès personnels : c'était un dandy, un homme public faiseur de modes, un homosexuel cultivant le scandale avec délice. Il était en quête d'intensité dans sa vie morale et affective, jusqu'à se mettre en danger (il est mort à 51 ans, usé par toutes sortes d'excès, notamment la consommation d'éther) : "Pour éprouver une émotion nouvelle, il n'est rien qu'il ne sacrifie. Il ne s'est refusé aucune des sensations agréables ou étranges. Il a surmené son corps et son âme ${ }^{19}$. » Ces émotions, dont l'expérience est un gage de vie réellement vécue, se manifestent chez lui de façon paroxystique, ainsi qu'il l'analyse lui-même dans le cadre intime d'un échange épistolaire avec son ami Octave Uzanne : «Je ne puis regarder attentivement la vie sans éclater de rire, mais quand je ris aux éclats, je souffre abominablement. C'est ma façon de pleurer à moi, qui n'ai pas les yeux faits comme tout le monde ${ }^{20}$ » (nous soulignons). On y retrouve l'ambivalence touchante du personnage : Lorrain minimise avec panache l'aveu pathétique de sa souffrance par l'affirmation de la profonde singularité de son émotivité, revendiquée non sans fierté comme idiosyncrasique.

Il ne s'agit pas ici de faire de la psychocritique (même si on ne peut s'empêcher de chercher l'auteur derrière son personnage de prince russe Noronsoff ${ }^{21}$ ), mais de situer Lorrain dans l'esthétique fin-de-siècle, notamment l'esthétique dandy, qui ne dissocie pas

16 Cité dans dans l'article «Barbares» du Dictionnaire du romantisme, op. cit., p. 64.

17 Le nom entre dans les dictionnaires de langue française dans les années 1830. Pour plus de détails, nous nous permettons de renvoyer à notre ouvrage (2015) : L'Imaginaire du mot "slave » dans les langues française et allemande, entre dictionnaires et romans. Paris : Editions Petra, pp. 142-144.

18 Michelet, J. (1974) : Le Peuple. Paris : GF Flammarion, p. 72.

19 L'affirmation est d'Achille Ségard, dans Les Voluptueux et les hommes d'action (1900), cité par Anthonay, T. de (1991) : Jean Lorrain barbare et esthète. Paris : Plon, p. 173.

20 Cité par Anthonay, T. de, ibid., p. 170.

21 Selon Philippe Jullian, ce roman « est peut-être le seul exemple d'une autobiographie imaginaire tendant vers la catastrophe ». Jullian, P. (1974) : Jean Lorrain ou le Satiricon 1900. Paris : Fayard, p. 275. 
la vie et l'œuvre, dans la mesure où l'esthète est appelé à faire œuvre de sa vie même. En ce sens Lorrain est lui-même un des acteurs de la décadence, jusque dans ses contradictions internes, qui sont aussi celles de la modernité marquée par un sentiment de scission interne : Antoine de Baecque le qualifie ainsi de « débauché consterné par la débauche du monde $^{22}$ ». Il y a de fait chez lui un moraliste ${ }^{23}$ qui pointe les travers de la mondanité, les ambitions sociales, le pouvoir de l'argent.

Il n'est donc pas anodin, pour notre étude de l'imaginaire de la barbarie dans Les Noronsoff, de relever que Lorrain lui-même a été qualifié de "barbare ", ainsi qu'Henry Bataille l'écrit dans La Renaissance latine en 1902 :

C'est une sorte de grand barbare, un barbare authentique, installé dans l'Urbs boulevardière, où il apporte et prodigue depuis vingt ans ses instincts de sang et de volupté, sa compréhension raffinée de la ville, son sens des ironies locales, sa politique madrée d'Oriental ou de Celte (car on ne saurait discerner au juste son origine réelle), et mêlant à cela, au goût des arts et de la culture, les brutalités les plus solitaires ou les plus criminelles. [...] Du barbare, il a la convoitise gourmande et l'amusement artiste, et aussi une sensibilité d'enfant très douce, facilement en larmes $[\ldots]^{24}$

Cette longue citation, ici réduite, rassemble des traits épars, ce qui pourrait étonner si Roger Pol-Droit ne remarquait justement que les discours relatifs aux barbares s'additionnent plus qu'ils ne s'annulent. Cette superposition de sèmes constitutifs dessinent un portrait syncrétique du barbare, mêlant raffinement, candeur et sauvagerie : son origine ethnique reste partiellement indéterminée, extérieure (orientale) et/ou intérieure (celte). Il laisse libre cours à des émotions variées et contradictoires, exprimées de façon désordonnée et impulsive. Il est à la fois enfant, donc lié à un monde originel où s'expriment sans distance des émotions spontanées, et esthète, c'est-à-dire hautement civilisé. En effet l'émotion esthétique, spécialement celle que suscite un art éloigné du réel et de sa laideur, permet à l'homme désabusé des civilisations avancées, décrit par Chateaubriand, de lutter contre la désillusion et le dégoût de vivre. Cette même désillusion héritée du romantisme est au cœur des Noronsoff, où l'on retrouve ce syncrétisme de la barbarie.

\section{Barbarie et syncrétisme fin-de-siècle dans Les Noronsoff}

Les Noronsoff est le long récit publié, avec d'autres nouvelles sous-titrées «Coins de Byzance ", dans Le Vice errant. L'action est située dans le cadre cosmopolite de la Riviera, à Nice (où Lorrain lui-même s'est installé dans les dernières années de sa vie avec sa mère). C’est le décor de la décadence par excellence, où se retrouvent « tous les déséquilibrés et tous les hystériques ${ }^{25}$ » de la terre. Le personnage principal, Wladimir Noronsoff,

22 Lorrain, J. (2015) : Souvenirs d'un buveur d’éther, éd. par Baecque, A. de. Paris : Mercure de France, "Le Temps retrouvé », p. 17.

23 Sur la complexité de cette posture, voir l'article de Záviš Šuman (2015) : "Instabilité dans l’imaginaire de Jean Lorrain : échos et reflets dans Monsieur de Phocas ", Acta Universitatis Carolinae, Philologica 1/2015, Romanistica Pragensia XX, pp. 41-47.

24 Cité par Kyria, P. (1973) : Jean Lorrain. Paris : Seghers, p. 121.

25 Lorrain, J. (1980) : Maschere. In Le Vice errant. Paris : J.-C. Lattès, « Les Classiques interdits », p. 65. 
est un prince russe richissime et " dégénéré », un "instinctif surmené de jouissances ${ }^{26}$ », marqué par le double atavisme de la race : la lignée maternelle florentine serait issue d'Alexandre Borgia et la lignée paternelle slave souffre d'une malédiction ancestrale lancée par une bohémienne, qui aliène sexuellement ses victimes. Le roman, dans ses réjouissants excès carnavalesques, ne s'interdit aucune provocation, à l'image du prince qui se plaît à inventer sans cesse de nouveaux caprices propres à choquer la société niçoise qui le marginalise. Ainsi, dans un chapitre dont le titre fait écho au Satiricon de Pétrone, "Le souper de Trimalcion ", il présente à ses invités, sur un plateau, des pêcheurs du port, nus et tatoués. Il est en outre flanqué d'une Polonaise ruinée, Vera, une aventurière perverse et coquette, qu'il prend plaisir à humilier. Il y a donc une double slavité dans le roman, entrelacée à la judéité dans le cas de Vera. Le roman reflète l'idéologie racialiste de l'époque puisque la race y est présentée comme le principe explicatif des comportements. Noronsoff est très souvent désigné comme "le Russe ", mais il est aussi qualifié de cosaque ou tartare, ce qui appelle à replacer le nom " slave » dans un paradigme oriental, lequel présuppose le raffinement dans la cruauté, la sensualité débridée, le despotisme (visible notamment dans la compétition de servitude et de domination à laquelle se livrent le Russe et la Polonaise). Il y a donc une racialisation du régime émotionnel des individus, illustré dans les excès auxquels ils se livrent.

Cette slavité est bien identifiée comme «barbare », terme qui vient très fréquemment qualifier le prince, mais le plus souvent accolé à l'adjectif « raffiné ». Comme le suggérait la référence à Pétrone précédemment citée, on retrouve ici une conception complexe de la barbarie, hautement civilisée, et en outre syncrétique, dans la mesure où, dans Les Noronsoff, elle conduit à mêler à la fascination slave un imaginaire bas-latin, typique de l'époque. Rappelant l'engouement pour le modèle de la décadence romaine à la fin du XIX ${ }^{\mathrm{e}}$ siècle en Europe ${ }^{27}$, Marie-France David de Palacio qualifie le roman de "plus romain que russe ", Noronsoff y étant assimilé à un empereur décadent, un Néron « slavisé » (Néron-soff). Elle montre l'abondance de lieux communs de cet imaginaire antiquisant fin-de-siècle : le prince se livre à des caprices dignes des empereurs romains, et cherche dans l'Antiquité les sources du Beau. Il reconstitue par exemple une scène mythologique (les funérailles et la résurrection d'Adonis), avec des dizaines de figurants venus du peuple. Cette fête engloutit une partie de sa fortune et suscite une fièvre qui « contamin $[\mathrm{e}]$ » la ville entière, en proie à « la manie érotique et fastueuse du prince ${ }^{28}$ ". La référence romaine ici indique le caractère paroxystique, déséquilibré, de l'émotion esthétique recherchée par le décadent blasé. Elle ravive en outre historiquement le souvenir d'une fragilisation politique de l'Empire romain, qui renvoie les contemporains à leurs propres inquiétudes face à l'intégrité de leur territoire. Désireux de trouver un soutien contre l'ennemi allemand, les Français se sont tournés vers les Russes, avec qui a été nouée une alliance dans les années 1890. Mais ce rapprochement n'est pas sans susciter certaines craintes, d'autant que la Russie est mal connue et perçue comme lointaine : craintes dont on pourrait retrouver une trace dans le roman de Lorrain, où le caractère

26 Lorrain, J. (2002) : Les Noronsoff. Paris : La Table ronde, « La petite vermillon », p. 44.

27 David-de Palacio, M-F. (2009) : «Coins de Rome... et de Byzance : la référence à l'antique chez Jean Lorrain ». In Palacio, J. de - Walbecq, E. Jean Lorrain. Produit d'extrême civilisation. Mont-Saint-Aignan : PURH, pp. 43-59.

28 Les Noronsoff, op. cit., p. 184. 
slave apparaît dangereusement versatile et peu fiable. Il est d'autant plus inquiétant qu'il prend aussi le visage du féminin et, surtout, d'une féminité juive, donc d'une altérité intérieure que les déchaînements antisémites de l'époque désignent toujours plus clairement comme ennemi du dedans. À l'échelle européenne, le sentiment de décadence et de crise de la civilisation est également formalisé au tournant du siècle sous la forme d'un mythe, celui du « péril jaune » venu d'Extrême-Orient.

\section{Le « péril jaune » : peur ou jouissance?}

Ce mythe du « péril jaune » trouve lui aussi des racines dans l'imaginaire bas latin. Il est décliné de façon très concrète à travers plusieurs scènes spectaculaires, situées à la fin du roman, comme pour mieux signifier le sens inéluctable de l'Histoire. De nouveau se trouvent mêlées les provenances des envahisseurs : Latins dans le premier cas, Asiatiques dans le second. La première scène est empreinte d'une confusion très babélienne : alors que les fêtes d'Adonis ont dû être interrompues en raison de la trahison de $\operatorname{Vera}^{29}$, la colère gronde dans les jardins, sous les fenêtres du prince, victime d'une crise d'apoplexie. Les figurants hystériques réclament leur dû à grands cris, en " patois » ou " jargon » niçois, napolitain, piémontais : "cétait à la fois l'atmosphère de rut d'un soir de festin et celle d'une insurrection populaire ${ }^{30}$. »Cette menace barbare, qui réactive ici une iconographie du peuple en colère à Versailles, combine le double sens ethnique et social de la barbarie. La deuxième scène illustre pour sa part très explicitement le "péril jaune », lors de l'agonie «furieuse » et « grandiose» du prince :

Halluciné, tordu de spasmes et d'épouvante, il appelait les Asiatiques et leur future invasion, leur trombe vengeresse sur la décomposition du vieux monde. [...] ses instincts barbares enfin réveillés conviaient les Barbares au châtiment. Il réclamait les Huns d’Attila et les Tartares de Genghis-Khan, toutes les hordes des races jaunes pour tuer, piller, voler, massacrer les Niçois, les médecins et Gourkau et lui-même, et sa mère. [...] Et dans un hoquet suprême il crachait enfin la vieille âme de Byzance trop longtemps attardée en luii ${ }^{31}$.

La peur collective exprimée dans ce mythe s'inscrit aussi dans l'imaginaire racialiste de l'époque. De fait le péril jaune reflète la hantise d'une perte de la supposée pureté raciale, au contact de l'étranger. Cet imaginaire racialiste est cependant pris dans une aporie, dans la mesure où la pureté raciale ne peut qu'être comprise comme utopie par ses tenants mêmes : l'idéologie de la décadence prend source dans les thèses de Gobineau, qui, dans son célèbre Essai sur l'inégalité des races humaines (1853), prend déjà acte de l'inéluctable métissage en cours, source précisément de la décadence constatée. Noronsoff n'est-il pas lui-même un produit du métissage, un être pour qui la barbarie est à la fois extrinsèque et intrinsèque, ainsi que le note le narrateur, en évoquant ses « instincts barbares enfin réveillés » ? Des indices de sa nature asiatique, sur un mode allusif

29 Alors que le prince a imaginé ce spectacle pour impressionner Vera, elle lui fait faux bond au dernier moment.

30 Les Noronsoff, op. cit., p. 187-188.

31 Ibid., p. 233. 
donc d'autant plus suggestif et pernicieux (comme la fente inquiétante de ses yeux), ont été auparavant disséminés dans le texte. C'est bien une civilisation " fin-de-race ", déjà contaminée par la barbarie (à l'image de Néron, civilisé mais ô combien barbare) qui, à travers lui, expire dans un déchaînement d'autodestruction jubilatoire. Noronsoff lance un appel provocateur à ce déferlement étranger : mais cette destruction peut être synonyme de salut pour une Europe à bout de souffle, que la vitalité du sang barbare viendrait régénérer, lançant le début d’un nouveau cycle.

\section{Barbarie, slavité et métamorphose}

C'est bien à l'échelle de l'Europe, une Europe se présentant comme l'incarnation de l'idée de civilisation, que se jouent ces questionnements identitaires. Confronté à l'hybridité complexe du personnage, on est amené à se demander si la slavité du personnage est, dans l'ordre des représentations, encore déterminante et fondatrice de sa barbarie. Noronsoff est russe, italien, tartare, cosaque, asiatique. Il est même, plus étonnant sans doute, "nègre ", voire "vieille négresse ", ainsi que le dévoile le récit de métamorphose du prince lisant la lettre assassine de la Polonaise. Le caractère physiologique des émotions y est patent, mettant en jeu une « rhétorique de la peau ${ }^{32}$ » :

[...] ç'avait été une chose terrifiante : toute la face du Russe s'était comme rapetissée, les yeux singulièrement amincis ne laissant plus filtrer qu'une lueur d'or, les traits crispés et ratatinés comme ceux d'un très vieux visage, dont toutes les rides se fussent creusées à la fois. Le prince avait lu en silence, puis l'étonnante face de momie s'était tout à coup obscurcie; de lie de vin elle était devenue violette, telle une aubergine ; puis, ç'avait été une tête de nègre, de vieille négresse plutôt avec des yeux chavirés et blancs ${ }^{33}$.

Cette surprenante métamorphose, comique dans son outrance, loin d'annuler le principe « racialiste » de slavité à l’ouvre dans le comportement du prince, serait une ultime illustration de la supposée essence slave : une essence marquée par le manque, par un déficit ontologique et historique, source d'une propension à l'imitation - ce discours se retrouve aussi dans le champ esthétique, où les Slaves sont qualifiés de « race imitatrice ${ }^{34}$ ». À l'échelle européenne, et dans ce contexte de théorisation du mythe (racial et linguistique) indo-européen, ils endossent un rôle ambigu en tant qu' " Aryens orientaux ", dans un entre-deux. Le "peuple» russe est par exemple souvent décrit comme un peuple de race impure car très métissé. Les Slaves, incarnant l'altérité intérieure, peuvent désormais être un élément déclencheur de la fonction barbare « dehors de $\mathrm{X}$ » mise en lumière par Roger Pol-Droit, laquelle n'indique plus tant la séparation avec l'extérieur que le surgissement de la scission en soi.

\footnotetext{
Voir l'article de Pascale Auraix-Jonchière dans ce même volume, page 108.

Ibid., p. 199.

4 Ainsi dans De l'Allemagne de $\mathrm{M}^{\mathrm{me}}$ de Staël. Voir Michaud, E., op. cit., p. 118.
} 


\section{Le désordre des émotions : une pathologie fin-de-siècle?}

Ces « barbares slaves » incarnent donc, plus que d'autres, le déséquilibre émotionnel interne à la civilisation. Dans le chapitre intitulé "L’âme russe ", ce désordre pathologique est dévoilé dans des chants populaires, qui, héritage romantique oblige, sont reçus comme l'expression innée et authentique du Volksgeist. De fait Noronsoff, après avoir manqué mourir une première fois, se console en écoutant chanter les moujiks employés aux cuisines. Ces chants désespérés dévoilent selon lui un profond malaise existentiel, enraciné dans une culture russe envisagée de façon essentialiste, d’où un malentendu indépassable entre "Russes » et « Européens » dont témoignerait justement l'accusation de barbarie :

Quelle voix ont ces misérables! Ils ne chantent pas, ils pleurent. [...] Nous autres Russes, les autres peuples ne nous comprennent pas ; est-ce que ma mère me comprend ! Je suis un étranger pour elle, nous sommes des barbares pour l'Europe ${ }^{35}$.

Le prince se livre alors à un exposé sur le déséquilibre pathologique de «l'âme russe ». Mais la maladie "nationale » dont souffriraient ses compatriotes éveille chez le lecteur des échos pour le moins familiers : «L'ennui ! Étrange maladie, désarroi nerveux, spleen chronique qui pénètre chez nous jusque dans les masses profondes du peuple ${ }^{36}$. » Cette maladie de l'âme, que les romantiques et leurs successeurs tout au long du XIX ${ }^{e}$ siècle ont cherché à nommer, n'est-elle pas celle de l'homme moderne, très, voire trop, civilisé ? Une fois de plus, et malgré l'artifice du dispositif prêtant au Russe un discours identitaire fondé sur la clôture, les lignes entre le barbare et le civilisé se défont dans la circulation d'un imaginaire commun - ce Russe est d'ailleurs mâtiné de "vieille civilisation » italienne. Il serait donc plus juste de qualifier Noronsoff de "barbare civilisé », en insistant sur le singulier qui, à l'encontre du pluriel couramment employé pour les barbares, tend à le singulariser (du côté de la civilisation), mais à la façon d'un hapax, d'un cas particulier car pathologique (ou pathologique car particulier). La vie intérieure du prince est déréglée, ce qui se traduit physiologiquement dans son corps malade, quasi pourrissant.

Il faut replacer l'étude des émotions dans leur contexte historique : en cette fin de siècle, qui voit la psychologie s'imposer, la vie intérieure est perçue comme de plus en plus complexe, donnant naissance à des théorisations scientifiques mais aussi à des formes artistiques - mentionnons par exemple la vogue du roman psychologique à la Paul Bourget. Le corps médical prête une attention aiguë aux manifestations des émotions, a fortiori sous leur forme excessive, déréglée, si ce n'est monstrueuse. Ce n'est pas un hasard que le narrateur du roman de Lorrain soit un médecin. Il élève le cas particulier à une loi générale, révélatrice du caractère biologique de la psychologie des peuples en plein essor. L'observation clinique de Noronsoff, épris de sa propre souffrance qu'il considère avec orgueil et curiosité, permet d'expérimenter et de confirmer ces théorisations : « je retrouvais $[\ldots]$ toute l'âme à la fois veule et passionnée de Wladimir, cette manie du

35 Les Noronsoff, op. cit., p. 206.

36 Ibidem. 
dédoublement et cet égoïsme voluptueux s'aiguisant dans l'analyse, qui est la caractéristique de l'âme russe ${ }^{37}$."

« Âme » russe ? Ou « âme » occidentale contemplant sa propre émotion dans celle de l'autre, et se découvrant pareillement scindée ? Le roman illustre à la fois cette fascination nouvelle pour l'analyse de soi et la peur face à des flux émotionnels non canalisés, qui prennent la forme d'un débordement étranger. Cette inquiétude est néanmoins plus le fait de ses contemporains que de Lorrain lui-même, qui réinvestit de façon ludique le riche matériau romanesque que constitue le mythe de la slavité, pour en donner sa propre version fin-de-siècle. Les Noronsoff peuvent donc se lire comme l'expression finalement très intime des excès émotionnels collectifs.

La slavité permet bien d'illustrer la fluctuation, évoquée en introduction, entre les pôles civilisé et barbare. La barbarie slave conjoint en effet les deux versants, extérieur et intérieur, de la barbarie : mythe des invasions étrangères, et intériorisation de la barbarie. Elle donne forme au sentiment angoissé de fin de cycle et à la peur du métissage. Mais la menace barbare est aussi porteuse d'un remède à ce sentiment de décadence : en invitant à laisser libre cours aux affects, à féconder l'art par le rêve et la déraison (par exemple dans le symbolisme), la «barbarisation » de l'art, combattu par Maurras et les tenants d'un patriotisme littéraire, est plébiscitée par bon nombre d'artistes qui ne craignent pas de décentrer leurs références et leurs modèles. Chez Lorrain, que le déséquilibre n'effraie pas, la crainte suscitée par ces invasions barbares va jusqu’à se renverser en jouissance. On reconnaît là la posture provocatrice de l'écrivain, mais il faut aussi y voir le signe d'une place faite à l'étranger comme à un autre soi-même, la peur laissant place à la pitié, sème constitutif du cliché de l'âme russe. C'est ainsi que Le Vice errant, dans lequel vient s'insérer l'histoire du prince, se voit qualifié dans la dédicace de « livre de pitié38 ", exprimée envers ce marginal déclassé et pathétique qu'est Noronsoff, ce barbare civilisé qui meurt d'avoir trop voulu vivre.

\section{BIBLIOGRAPHIE}

Anthonay, T. de (1991) : Jean Lorrain barbare et esthète. Paris : Plon.

Brogniez, L. (2001) : Le mythe de l'invention des barbares en Belgique : art ou/et révolution au tournant du siècle ». In Montandon, A. Mythes de la décadence. Clermont-Ferrand : Presses Universitaires Blaise Pascal, pp. 143-162.

Corbin, A. - Courtine, J.-J. - Vigarello, G. (2016-2017) : Histoire des émotions. Paris : Seuil.

David de Palacio, M.-F. (2009) : «Coins de Rome... et de Byzance : la référence à l'antique chez Jean Lorrain ». In Palacio, J. de - Walbecq, E. Jean Lorrain. Produit d'extrême civilisation. Mont-Saint-Aignan : PURH, pp. 43-59.

Guirlinger, L. - Poly, J.-P. - Lefort, C. - Mijolla-Mellor, S. de - Mattéi, J.-F. - Nahoum-Grappe, V. Brossat, A. (2005) : La barbarie aujourd'hui, mythe et réalité. Nantes : Éditions Cécile Defaut.

Jullian, P. (1974) : Jean Lorrain ou le Satiricon 1900. Paris : Fayard.

Kyria, P. (1973) : Jean Lorrain. Paris : Seghers.

Lorrain, J. (1980) : Le Vice errant. Paris : J.-C. Lattès, « Les Classiques interdits ».

37 Les Noronsoff, op. cit., p. 204.

38 Le Vice errant, op. cit., p. 15. 
Lorrain, J. (2002) : Les Noronsoff. Paris : La Table ronde, « La petite vermillon».

Lorrain, J. (2015) : Souvenirs d'un buveur d'éther, éd. par Baecque, A. de. Paris : Mercure de France, «Le Temps retrouvé ».

Michaud, E. (2015) : Les Invasions barbares. Une généalogie de l'histoire de l'art. Paris : Gallimard, «nrf essais $"$.

Michel, P. (1981) : Un mythe romantique. Les Barbares 1789-1848. Lyon : Presses universitaires de Lyon.

Pol-Droit, R. (2007) : Généalogie des barbares. Paris : Odile Jacob.

Rey, M-P. (2015) : Un tsar à Paris. 1814. Alexandre I er et la chute de Napoléon. Paris, Flammarion, "Champs".

Said, E. (2013) : L'Orientalisme : l'Orient créé par l'Occident, trad. de l'américain par C. Malamoud. Paris : Points, « Essais».

Šuman, Z. (2015) : «Instabilité dans l'imaginaire de Jean Lorrain : échos et reflets dans Monsieur de Phocas », Acta Universitatis Carolinae, Philologica 1/2015, Romanistica Pragensia XX, pp. 41-47.

Vaillant, A., dir. (2012) : Dictionnaire du romantisme. Paris : CNRS Editions.

Voldřichová Beránková, E. - Grauová, Š. (2017) : Dusk and Dawn. Literature between Two Centuries. Prague : Univerzita Karlova, Filozofická Fakulta.

\section{Cécile Gauthier}

URCA, Département de Lettres modernes (Littérature comparée)

UFR Lettres et Sciences Humaines

57 rue Pierre Taittinger

51096 Reims Cedex

cecile.gauthier@univ-reims.fr 